\title{
Indicações para bloqueios anestésicos guiados por ultrassom na emergência: Uma revisão narrativa
}

\author{
Indications to ultrasound-guided nerve blocks in the emergency: A narrative review
}

Indicaciones para bloqueos anestésicos guiados por ultrasonido en la emergencia: Una revisión narrativa

Rodrigo Luiz Damázio de Oliveira ${ }^{1 *}$, Adriano Aragão da Rocha ${ }^{1}$, Rebeca de Souza Mariano Bastos ${ }^{1}$.

\section{RESUMO}

Objetivo: Fornecer a médicos emergencistas exemplos de aplicações bem-sucedidas de bloqueios nervosos guiados por ultrassom relatadas na literatura. Revisão Bibliográfica: Dos artigos nesta revisão incluem 11 (onze) estudos prospectivos (7 com amostras de conveniência e 4 randomizados), 1 estudo de coorte, 1 revisão sistemática e 19 relatos de casos, únicos ou em série. Didaticamente, o conteúdo dos estudos sobre bloqueio guiado por US foi descrito de maneira narrativa em forma de indicações categorizadas de acordo com a porção do corpo acometida pela intervenção, grupo populacional específico ou condições específicas com grande densidade de estudos, a saber: Lesão em extremidades superiores; Luxação de ombro; Fratura de arcos costais; Lesão em parede abdominal; Lesão de extremidades inferiores; Fraturas de quadril e colo de fêmur; População pediátrica; Outras indicações. Considerações Finais: Esta revisão demonstra que bloqueios anestésicos guiados por ultrassom são viáveis no departamento de emergência e destaca que diminuição significativa e sustentada da dor pode ser alcançada com essa intervenção.

Palavras-chave: Bloqueio Nervoso; Ultrassonografia; Serviço Hospitalar de Emergência.

\begin{abstract}
Objectives: Give emergency physicians examples of successful applications of ultrasound guided nerve blocks in the literature. Literature Review: Among the articles included in this review, there are eleven (11) prospective studies ( 7 with convenience samples and 4 randomized studies), 1 cohort study, 1 systematic review, and 19 single or serial case reports. In a didactic way, the content of ultrasound guided nerve block studies was narratively described as several indications categorized according to the body portion affected by the intervention, specific population group affected, or specific conditions with high density of studies shown in the literature, to acknowledge: Injury of superior limbs; Shoulder dislocation; Fracture of costal arches; Abdominal wall injury; Injury of lower extremities; Hip and femoral neck fractures; Pediatric population; Other indications. Final Considerations: This review demonstrates that us guided nerve blocks are viable in the emergency department and highlights that significant and sustained decrease of pain can be achieved by this intervention.
\end{abstract}

Keywords: Nerve Block; Ultrasonography; Emergency Service, Hospital.

\section{RESUMEN}

Objetivos: Proporcionar a médicos emergencistas ejemplos de aplicaciones bien sucedidas de bloqueos nerviosos guiados por ultrasonido relatadas en la literatura. Revisión Bibliográfica: Los artículos en esta revisión incluyen once (11) estudios prospectivos (7 con conveniencia y 4 asignados al azar), 1 estudio de

${ }^{1}$ Universidade Tiradentes (UNIT), Aracaju-Sergipe. *E-mail: damaziorodrigo.Ido@gmail.com 
cohorte, 1 revisión sistemática y 19 informes de casos individuales o en serie. Didácticamente, el contenido de los estudios de bloqueos guiados por ultrasonido se describió narrativamente como indicaciones clasificadas de acuerdo con la porción del cuerpo afectada por la intervención, el grupo de población específico o las condiciones específicas con alta densidad de estudio, a saber: lesión en la extremidad superiores Luxación de hombro; Fractura de arcos costales; Lesión de la pared abdominal; Lesión de la extremidad inferior; Fracturas de cadera y cuello femoral; Población pediátrica; Otras indicaciones. Consideraciones Finales: Esta revisión demuestra que bloqueos anestésicos guiados por ultrasonido son viables en el departamento de emergencia y destaca que disminución significativa y sostenida del dolor puede ser alcanzada con esa intervención.

Palabras Ilave: Bloqueo Nervioso; Ultrasonido; Servicio Hospitalario de Emergencia.

\section{INTRODUÇÃO}

Dor é a principal queixa de pacientes em diversos departamentos de emergência (DE) pelo mundo. Além disso, muitos procedimentos de emergência como redução de fraturas causam dor e requerem analgesia eficiente a fim de ganhar cooperação do paciente durante o procedimento (CORDELL WH, et al., 2002). Apesar de muitos médicos prescreverem opioides sistêmicos nesses casos, nem toda dor é tratada adequadamente e, em alguns casos, esses medicamentos podem trazer efeitos deletérios, sendo não apenas ineficazes, mas contribuindo para o abuso e uso indevido (DUNCAN RW, et al., 2019).

A sedação processual oferece aos pacientes conforto e relaxamento muscular para facilitar procedimentos dolorosos, contudo, há riscos associados incluindo hipotensão, broncoaspiração, depressão respiratória, entre outros. Tais riscos são minimizados por protocolos padrão em vários DEs que requerem monitorização cardíaca, de pressão arterial, saturação de oxigênio e capnografia (BLAIVAS M, et al., 2011; ROBACK MG, et al., 2005). No entanto, isso requer cuidados "um-a-um" da enfermagem e pode requerer o uso de instalações de cuidado crítico ou de maior complexidade dentro do DE. Já que a maioria dos DEs não são designados para monitorização completa e suporte de via aérea em cada leito, o manejo adequado da dor torna-se pouco viável nesse ambiente e pacientes podem precisar ser realocados.

Essa situação induziu médicos do DE a procurarem métodos alternativos à sedação e ao uso de opioides sistêmicos. Bloqueios regionais como supraclavicular, plexo braquial e lidocaína intra-articular tem sido usado em procedimentos dolorosos como redução de fraturas e limpeza exaustiva de feridas extensas (TEZEL O, et al., 2014). Anestesia regional é frequentemente usada como adjunta à anestesia geral para cirurgias ortopédicas, já sendo bem difundida e consolidada no ambiente perioperatório. Bloqueios nervosos regionais tem sido realizado tradicionalmente às cegas e requerem equipamento especializado como estimuladores de nervo que não são tipicamente encontrados no DE (BLAIVAS M, et al. 2011; DAVIS JJ, et al., 2009). No entanto, orientação por ultrassom (US) está se tornando uma técnica cada vez mais popular a facilitar bloqueios nervosos regionais na prática anestésica. Recentemente, múltiplos relatórios apareceram descrevendo o uso de bloqueios nervosos guiados por US por médicos emergencistas, o que pode conferir vantagens significantes sobre sedação processual e uso de opioides sistêmicos em diversos DEs (DAVIS JJ, et al., 2009).

A ultrassonografia tem revolucionado a habilidade de emergencistas em integrar bloqueio de nervos periféricos a uma abordagem multimodal de manejo da dor no $\mathrm{DE}$, tornando esses profissionais cada vez mais familiarizados com essa modalidade de imagem. Há evidências mostrando que bloqueios de nervos periféricos guiados por ultrassom são realizados com menor quantidade de anestésico e maiores taxas de sucesso, tendo impacto positivo sobre o tempo de estadia no leito de emergência, menor necessidade de monitorização contínua e suporte de via aérea, oferecendo também um melhor desfecho em relação a complicações precoces e tardias (LAPIETRA AM, et al., 2016).

Bloqueios nervosos guiados por ultrassom foram introduzidos em anestesiologia em 1978, quando La Grange et al utilizou um dispositivo Doppler para realizar um bloqueio supraclavicular do plexo braquial. No 
entanto, foi no fim de 1994 que o ultrassom foi usado a fim de mostrar a exata localização do nervo e a área perineural onde o anestésico seria injetado (BHOI S, et al., 2010; LA GRANGE PP, et al., 1978).

$\mathrm{Na}$ maioria dos hospitais, médicos anestesiologistas são os mais qualificados a performar bloqueios nervosos. No entanto, devido a demandas de trabalho e restrição de tempo, esses profissionais podem não estar aptos a atender pacientes no departamento de emergência ou unidade intensiva em tempo hábil, levando a atrasos significativos em promover analgesia de qualidade (BLAIVAS M, et al., 2011). Randall et al reportou os resultados de uma iniciativa bem-sucedida de capacitar enfermeiros para realizar bloqueio de fáscia ilíaca. Essa criação de uma "extensão do médico" melhorou o acesso do paciente ao controle efetivo da dor utilizando um procedimento simples e seguro que é facilmente ensinado (BLAIVAS M, et al., 2011; RANDALL A, et al., 2008).

O objetivo do presente estudo é fornecer uma revisão bibliográfica direcionando a médicos emergencistas exemplos de aplicações bem-sucedidas de bloqueios nervosos guiados por ultrassom relatadas na literatura. Com esse propósito almejamos comprovar, mesmo que não definitivamente, o valor dessa técnica e consequentemente expandir o seu uso dentro dos DEs. Com a disseminação dessa modalidade de manejo da dor, esperamos que novos e maiores estudos sejam construídos para corroborar essa comprovação.

\section{REVISÃO BIBLIOGRÁFICA}

\section{Lesão em Extremidades Superiores}

No estudo prospectivo feito em pacientes com lesões de mão, os bloqueios guiados por ultrassom de nervos radial, ulnar e mediano puderam ser realizados no pronto-socorro com sucesso. Em $100 \%$ dos casos não foi necessária analgesia adicional após o bloqueio; A redução média no escore visual analógico da dor após 15 minutos foi de 5 milímetros. Não foram relatadas complicações imediatas (punção nervosa ou intravascular) ou tardias durante os 3 meses de acompanhamento (LIEBMANN O, et al., 2006).

Um relato de caso em 2018 mostrou o sucesso de bloqueio de nervo ulnar guiado por ultrassom em paciente com neuropatia ulnar secundária a vasculite e compressão por fístula arteriovenosa, apresentando dor severa em antebraço. O procedimento proporcionou completa satisfação do paciente com resolução completa da dor por 10 meses (CANDERS CP, et al., 2018).

Na série de 4 (quatro) casos de fratura de rádio distal, o bloqueio de nervo radial por via supracondilar guiado por ultrassom foi provado efetivo. Foi observado significativa redução da dor no escore visual analógico (médias de 93,5 para 21), ausência de danos neurovasculares no acompanhamento por seis meses após procedimento, além da facilidade de ser realizado por apenas um operador (AYDIN AA, et al., 2016).

Em uma série de 4 (quatro) casos, pacientes no DE foram eleitos a receber bloqueio nervoso em região de antebraço após receberem múltiplas doses intravenosas de hidromorfona, morfina, fentanil, ketamina e/ou midazolam com analgesia ineficaz. Os bloqueios foram realizados por emergencistas, possibilitando a equipe de cirurgia avaliar a extensão de grandes lesões, fazer controle de hemorragia via clampeamento, irrigação exaustiva, sutura de lacerações complexas, redução de fraturas e aplicação de enxerto de pele sem causar dor ao paciente (WROE P, et al., 2016).

Quanto ao bloqueio de plexo braquial guiado por ultrassom, um estudo de 2015 apresentou a primeira descrição de um bloqueio infraclavicular realizado por emergencistas para redução de luxação de cotovelo. O homem de 29 anos com dor intensa em membro superior esquerdo e deformidade do olécrano foi submetido a uma redução sem dor do cotovelo 20 minutos após realização do bloqueio. Complicações como dispneia, pneumotórax ou paralisia não foram relatadas (HEFLIN T, et al., 2015).

Um estudo prospectivo em 2018 avaliou a aplicação de bloqueio de plexo braquial guiado por ultrassom em 27 pacientes com quadros de espasmos musculares paracervicais, fraturas de clavícula, lesões de articulação acromioclavicular, dor radicular e síndrome do manguito rotador. A redução nos escores da dor 
foi excelente em dez pacientes (variando, em média, de 9 para 1 após 5 minutos), pobre em 4 pacientes (Variando de 9 para 7, em média) e moderada no restante da amostra. Foram relatadas duas complicações precoces: paresia hemidiafragmática e paralisia de nervos frênico e laríngeo recorrente, com todas sendo resolvidas em um período de no máximo 24 horas (HO B e DE PAOLI M, 2018).

Um relato de caso mostra uma aplicação bem-sucedida de bloqueio de plexo cervical superficial na emergência, em um paciente com fratura de clavícula à direita. Após 20 minutos do procedimento, houve uma redução de 10 para 2 no escore numérico de dor sem complicações e com efeito durando 20 horas, necessitando de analgesia suplementar com anti-inflamatório não esteroidal (AINE) (HERRING AA, et al., 2012).

\section{Luxação de Ombro}

Luxação de ombro é a luxação mais comum encontrada na população do departamento de emergência (DE), ocorrendo em 0,5 - 1,7\% dos indivíduos e o procedimento de redução de ombro pode ser extremamente doloroso, necessitando de manejo da dor apropriado (BLAIVAS M e LYON M, 2006).

Em dois estudos (2006 e 2011) para avaliar o bloqueio de plexo braquial guiado por US por via interescalênica, sendo um estudo de viabilidade e um de comparação com sedação processual tradicionalmente utilizada para esse tipo de procedimento (BLAIVAS M, et al., 2011; BLAIVAS M e LYON M, 2006). No estudo de 2006, houve uma taxa de sucesso de $100 \%$ na redução da dor durante a redução em um período de 15-30 minutos após bloqueio (BLAIVAS M e LYON M, 2006).

No estudo comparativo, a média de tempo de estadia no DE foi significantemente mais alta no grupo de sedação processual (177.3 minutos), comparada ao grupo de bloqueio interescaleno guiado por US (100.3 minutos). O tempo "um-a-um" de cuidado foi 47,1 minutos em média para o grupo de sedação e 5 minutos para o grupo de bloqueio interescaleno guiado por US. A satisfação do paciente e escores de dor foram similares em ambos os grupos. Não houve diferenças significativas entre grupos a respeito de complicações como hipóxia ou hipotensão (BLAIVAS M, et al., 2011).

Em 2011, um estudo de comparação entre bloqueio de plexo braquial supraescapular guiado por US e sedação processual foi realizado. Nele, uma diferença estatisticamente significativa foi determinada entre os grupos de sedação e bloqueio em relação ao tempo gasto no hospital e redução efetiva da dor: 0 tempo médio desde o início do procedimento até a alta foi de 125 minutos no grupo de sedação contra 25 minutos no grupo do bloqueio supraclavicular. A média da escala analógica de dor nos pacientes do grupo de bloqueio foi 85 na chegada e 45 após o procedimento. Nenhum efeito colateral incluindo hematoma, dano neural e injeção intravascular foi observado no grupo de bloqueio (TEZEL O, et al., 2014).

$\mathrm{Na}$ comparação entre bloqueio de plexo braquial e sedação processual realizada em 2008, não houve efeitos adversos associados ao bloqueio nervoso supraclavicular de plexo braquial, havendo um evento apneico autolimitado que não precisou de oxigenação suplementar no grupo de sedação processual. Nenhum objeto necessitou de analgesia adicional no grupo de bloqueio de plexo braquial, enquanto todos do grupo de sedação processual necessitaram de agentes analgésicos adicionais (mais comumente morfina e fentanil) após o término do procedimento. O tempo médio de estadia no DE no grupo de bloqueio de plexo braquial foi 106 minutos, enquanto no grupo de sedação processual foi 285 minutos. O tempo médio de iniciação do procedimento no bloqueio de plexo braquial foi 20 minutos, enquanto na sedação processual foi 199 minutos. Não houve diferença significativa no escore de satisfação do paciente (STONE MB, et al., 2007; STONE MB, et al., 2008).

Dois casos relatados em 2011 em pacientes com dor no ombro e diagnósticos de luxação de ombro e capsulite adesiva mostraram a aplicação bem-sucedida de bloqueio de nervo supraescapular guiado por ultrassom. No caso da capsulite o paciente manteve redução significativa da dor e melhora da função do membro durante duas consultas de acompanhamento (10 dias e 14 semanas), enquanto o caso de luxação teve a dor reduzida 15 minutos após o bloqueio, possibilitando o processo de redução do ombro (HERRING AA, et al., 2011). 


\section{Fratura de Arcos Costais}

Uma série de casos sugere um maior papel para o bloqueio de plano serrátil anterior em tratamento da dor na fratura de arcos costais no DE. Essas fraturas são comuns (10-38\% dos traumas no DE) e dolorosas. Elevar a analgesia com BPSA pode ajudar a melhorar função pulmonar, evitar efeitos adversos e reduzir mortalidade. Os casos avaliados relatam analgesia efetiva com redução total da dor após 30 minutos do bloqueio (DURANT E, et al., 2017).

Além disso, em outro caso é mostrado a eficácia do BPSA em uma paciente idosa com fratura de arcos costais refratária a analgesia tradicional com diclofenaco, acetaminofeno e tramadol IV. Após 15 minutos do bloqueio, foi possível à paciente se mover, se vestir e ser submetida a fisioterapia respiratória devido à redução significativa da dor (KUNHABDULLA NP, et al., 2014).

Em uma abordagem alternativa, uma série de casos abordou o uso de bloqueio do plano do músculo eretor da espinha para fraturas posteriores de arcos costais. Todos os pacientes que apresentavam dor torácica severa por fratura posterior de arcos costais e receberam o bloqueio tiveram analgesia efetiva dentro de 30 minutos, restaurando a habilidade de inspirar profundamente, tossir, rir e movimentar-se com mínima dor (LUFTIG J, et al., 2018).

\section{Lesão em Parede Abdominal}

Em uma série de casos, todos os bloqueios de plano transverso abdominal guiado por US foram realizados na primeira tentativa e o tempo médio para completar o procedimento foi de 9,5 minutos. Em todos os casos houve manejo adequado da dor, analgesia ou anestesia de resgate não foram necessárias para nenhum paciente e não houve complicações (HERRING AA, et al., 2012).

Foi publicado em um estudo que respondia aos estudos de outro artigo e neste relato mostrou que a eficácia do bloqueio de plano transverso abdominal em uma paciente com pancreatite aguda e que apresentou resistência a opioide sistêmico e contraindicação a anestesia neuroaxial. O procedimento foi realizado duas vezes, bilateralmente, com uma redução de 8 para $3 / 10$ (Escore numérico de dor) e a paciente foi liberada no quinto dia sem complicações (LANDY C, et al., 2016).

\section{Lesão em Extremidades Inferiores}

A série de casos relatada mostrou a eficácia do bloqueio de nervo poplíteo em casos de fratura de calcâneo, tornozelo, abscesso e laceração plantar. Em todos os casos, a dor foi completamente resolvida entre 10-15 minutos após o bloqueio e durou o suficiente para realizar limpeza da pele e redução das fraturas. Nenhuma complicação foi relatada (HERRING AA, et al., 2011).

Um relato de caso de luxação traumática de patela mostra a eficácia de bloqueio de nervo femoral guiado por ultrassom. Na ocasião, o bloqueio proporcionou uma redução da dor de 8 para 0 no escore numérico de dor após 5 minutos de injeção, possibilitando a redução patelar sem causar dor ao paciente (EKSERT S, et al., 2017).

\section{Fratura de Quadril e Colo de Fêmur}

Em dois estudos paralelos, foi posto à prova o bloqueio de nervo femoral $3 \mathrm{em} 1$ guiado por ultrassom: em 2010, relatou uma série de casos em um estudo de viabilidade; em 2013, comparou a técnica 3 em 1 à analgesia tradicional baseada em opioides sistêmicos (BEAUDOIN FL, et al., 2010; BEAUDOIN FL, et al., 2013). No primeiro estudo, todos os participantes receberam o bloqueio após o pico de efeito da morfina, sustentando alívio após o período de ação da mesma, relatando $100 \%$ de sucesso na realização do procedimento, atingindo alívio da dor. A resposta da dor ao procedimento foi variável: três pacientes atingiram e sustentaram um escore de $0 / 10$ após o bloqueio e a média entre toda a amostra foi de 3/10. Dos 13 participantes do estudo, 10 não precisaram de nenhuma analgesia de resgate (BEAUDOIN FL, et al., 2010).

No segundo estudo não houve diferença significativa entre grupos a respeito de tempo de estadia, mas houve diminuição significativa da intensidade da dor em pacientes do grupo de bloqueio, enquanto os 
pacientes do grupo de analgesia tradicional não tiveram mudança. Comparando alívio da dor entre grupos, a resposta ao tratamento em quatro horas foi significativamente maior no grupo de bloqueio. Pacientes no grupo de bloqueio tiveram alívio da dor efetivo e sustentado durante o período de quatro horas, diferente do grupo de analgesia padrão, que não sustentou melhora clínica importante (BEAUDOIN FL, et al., 2013).

Em uma revisão sistemática da literatura construída em 2016, foi avaliada a aplicabilidade do bloqueio de nervo femoral em fraturas de quadril. Em relação à redução da dor segundo o escore visual analógico, oito de nove estudos concluíram que há um benefício igual ou superior com bloqueio regional, comparado a modelos tradicionais de analgesia. Já para avaliação da redução de opioides IV, cinco de seis estudos que utilizavam opioides parenterais como analgesia padrão demonstraram uma redução estatisticamente significativa no consumo de opioides com bloqueio regional. O relato de complicações foi altamente variável em diferentes estudos, porém nenhum deles relatou complicações fatais dos bloqueios (RITCEY B, et al., 2016).

Um estudo randomizado com 161 pacientes com fratura de quadril comparou o bloqueio de nervo femoral à analgesia tradicional. A função motora se mostrou melhor em pacientes de intervenção e o número de participantes relatando efeitos colaterais foi menor no grupo de intervenção. Além disso, pacientes de intervenção relataram menores escores de dor, persistindo até o terceiro dia pós-operatório (MORRISON RS, et al., 2016). Em outro estudo, o menor valor no escore de dor foi alcançado em 120 minutos, o que representa uma queda de $76 \%$ na dor logo antes do procedimento (HAINES L, et al., 2012).

\section{Pediatria}

Em um estudo de coorte foi concluído que o bloqueio de nervo femoral guiado por ultrassom para dor aguda em fratura de fêmur promove um intervalo 2 a 3 vezes maior entre o tratamento inicial e a dose subsequente de medicação em comparação a medicações sistêmicas isoladas, sugerindo fortemente maior duração da analgesia. O tempo médio entre o início do tratamento e a segunda dose de analgesia foi de duas horas no grupo de analgesia tradicional contra seis horas no grupo do bloqueio de nervo femoral. Pacientes tratados com o bloqueio, no geral, necessitaram de menos doses de analgésicos e intervenções da equipe de enfermagem (TURNER AL, et al., 2014).

E outro estudo prospectivo em crianças com lesões na mão, foram realizados bloqueios de nervos ulnar, radial e mediano com um tempo de procedimento médio de 75 segundos. Houve redução significativa no escore visual analógico da dor, além de ausência de complicações imediatas ou tardias (FRENKEL O, et al., 2015).

Por fim, teve também outro estudo onde foi relatado um caso bem-sucedido de injeção intra-articular de lidocaína para redução de ombro. A técnica foi guiada por ultrassom para injeção na articulação glenoumeral e promoveu resolução completa da dor alguns minutos após o procedimento (BRESLIN K, et al., 2014).

\section{Outras Indicações}

Um estudo prospectivo avaliou 36 pacientes submetidos a bloqueios de nervos ciático, axilar e femoral com diversas condições clínicas, chegando a uma redução nos escores de dor de mais de $75 \%$ com poucos efeitos colaterais. A avaliação da satisfação tanto de pacientes como dos profissionais foi de aproximadamente $80 \%$. Apesar de haver relatos de redução significativa de pressão arterial e frequência cardíaca, não houve importância clínica (NEJATI A, et al., 2017).

Em um relato de 2015, teve um caso de parafimose se beneficiou do bloqueio de nervo dorsal do pênis, tendo resolução completa da dor possibilitando a redução do prepúcio 15 minutos após o bloqueio, sem complicações. Isso sugere o bloqueio como potencialmente efetivo no ambiente de emergência para dor peniana aguda e procedimentos como redução de parafimose e priapismo, tendo como principais vantagens a visualização de estruturas e o menor perfil de complicações (FLORES S e HERRING AA, 2015).

Também em outro estudo foi descrito a aplicabilidade do bloqueio do nervo auricular magno guiado por ultrassom em casos de abscesso e laceração de orelha na emergência. Em ambos os casos, analgesia efetiva 
foi alcançada 15 minutos após o procedimento, possibilitando sutura de lobo de orelha e drenagem de pus no abscesso. Não houve relatos de complicações precoces ou tardias em ambos os pacientes (FLORES S e HERRING AA, 2016).

\section{CONSIDERAÇÕES FINAIS}

Esta revisão demonstra que bloqueios anestésicos guiados por ultrassom são viáveis no DE e destaca que a diminuição significativa e sustentada da dor pode ser alcançada com essa intervenção. O curto tempo necessário para completar o procedimento, menos complicações e tentativas únicas sugerem que médicos com acesso à US beira de leito podem efetivamente realizar esse procedimento no ambiente de cuidado agudo. Apesar de as indicações necessitarem de mais estudos que confirmem a eficácia e segurança dos bloqueios, esse estudo sugere que bloqueio anestésico guiado por ultrassom é um bom adjunto ou talvez uma alternativa para opioides sistêmicos e sedação processual.

\section{REFERÊNCIAS}

1. AYDIN AA, et al. Novel technique in ED: supracondylar ultrasound-guided nerve block for reduction of distal radius fractures. The American journal of emergency medicine, 2016; 34(5): 912-913.

2. BEAUDOIN FL, et al. Ultrasound-guided femoral nerve blocks in elderly patients with hip fractures. The American journal of emergency medicine, 2010; 28(1): 76-81.

3. BEAUDOIN FL, et al. A comparison of ultrasound-guided three-in-one femoral nerve block versus parenteral opioids alone for analgesia in emergency department patients with hip fractures: a randomized controlled trial. Academic Emergency Medicine, 2013; 20(6): 584-59.

4. BHOI S, et al. Ultrasound-guided nerve blocks in the emergency department. Journal of Emergencies, Trauma and Shock, 2010; 3(1): 82.

5. BLAIVAS M, et al. A prospective comparison of procedural sedation and ultrasound-guided interscalene nerve block for shoulder reduction in the emergency department. Academic Emergency Medicine, 2011; 18(9): 922-927.

6. BLAIVAS M, LYON M. Ultrasound-guided interscalene block for shoulder dislocation reduction in the ED. The American journal of emergency medicine, 2006; 24(3): 293-296.

7. BRESLIN K, et al. Ultrasound-guided intra-articular lidocaine block for reduction of anterior shoulder dislocation in the pediatric emergency department. Pediatric emergency care, 2014; 30(3): 217-220.

8. CANDERS CP, et al. Management of an Acute Exacerbation of Chronic Neuropathic Pain in the Emergency Department: A Case to Support Ultrasound-Guided Forearm Nerve Blocks. The Journal of emergency medicine, 2018; 55(6): 147-151.

9. CORDELL WH, et al. The high prevalence of pain in emergency medical care. The American journal of emergency medicine, 2002; 20(3): 165-169.

10. DAVIS JJ, et al. Interscalene block for postoperative analgesia using only ultrasound guidance: the outcome in 200 patients. Journal of clinical anesthesia, 2009; 21(4): 272-277.

11. DUNCAN RW, et al. Alternatives to opioids for pain management in the emergency department decreases opioid usage and maintains patient satisfaction. The American journal of emergency medicine, 2019; 37(1): 38-44.

12. DURANT E, et al. Ultrasound-guided serratus plane block for ED rib fracture pain control. The American journal of emergency medicine, 2017; 35(1): 197.

13. EKSERT S, et al. Ultrasound-Guided Femoral Nerve Blockage in A Patellar Dislocation: An Effective Technique for Emergency Physicians. The Journal of emergency medicine, 2017; 52(5): 699-701.

14. FLORES S, HERRING AA. Ultrasound-guided dorsal penile nerve block for ED paraphimosis reduction. The American journal of emergency medicine, 2015; 33(6): 863.

15. FLORES S, HERRING AA. Ultrasound-guided greater auricular nerve block for emergency department ear laceration and ear abscess drainage. The Journal of emergency medicine, 2016; 50(4): 651-655.

16. FRENKEL $O$, et al. Ultrasound-guided forearm nerve blocks in kids: a novel method for pain control in the treatment of hand-injured pediatric patients in the emergency department. Pediatric emergency care, 2015; 31(4): 255-259.

17. HAINES $L$, et al. Ultrasound-guided fascia iliaca compartment block for hip fractures in the emergency department. The Journal of emergency medicine, 2012; 43(4): 692-697.

18. HEFLIN T, et al. Ultrasound-guided infraclavicular brachial plexus block for emergency management of a posterior elbow dislocation. The American journal of emergency medicine, 2015; 33(9): 1324. 
19. HERRING AA, et al. The ultrasound-guided superficial cervical plexus block for anesthesia and analgesia in emergency care settings. The American journal of emergency medicine, 2012; 30(7): 1263-1267.

20. HERRING AA, et al. Ultrasound-guided distal popliteal sciatic nerve block for ED anesthesia. The American journal of emergency medicine, 2011; 29(6): 697.

21. HERRING AA, et al. Ultrasound-guided abdominal wall nerve blocks in the ED. The American journal of emergency medicine, 2012; 30(5): 759-764.

22. HERRING AA, et al. Ultrasound-guided suprascapular nerve block for shoulder reduction and adhesive capsulitis in the ED. The American journal of emergency medicine, 2011; 29(8): 963.

23. HO B, DE PAOLI M. Use of Ultrasound-Guided Superficial Cervical Plexus Block for Pain Management in the Emergency Department. The Journal of emergency medicine, 2018; 55(1): 87-95.

24. KUNHABDULLA NP, et al. Serratus anterior plane block for multiple rib fractures. Pain Physician Journal, 2014; 17(4): 553-555.

25. LA GRANGE PP, et al. Application of the Doppler ultrasound bloodflow detector in supraclavicular brachial plexus block. British Journal of Anesthesia, 1978; 50(9): 965-967.

26. LANDY C, et al. Another use of the ultrasound-guided transversus abdominis plane block in the ED. American Journal of Emergency Medicine, 2012; 4(30): 626-627.

27. LAPIETRA AM, et al. Alternatives to Opioids for Acute Pain Management in the Emergency Department: Part II. Emergency Medicine Reports, 2016; 37(20).

28. LIEBMANN O, et al. Feasibility of forearm ultrasonography-guided nerve blocks of the radial, ulnar, and median nerves for hand procedures in the emergency department. Annals of emergency medicine, 2006; 48(5): 558-562.

29. LUFTIG J, et al. Successful emergency pain control for posterior rib fractures with ultrasound-guided erector spinae plane block. The American journal of emergency medicine, 2018; 36(8): 1391-1396.

30. MORRISON RS, et al. Regional nerve blocks improve pain and functional outcomes in hip fracture: a randomized controlled trial. Journal of the American Geriatrics Society, 2016; 64(12): 2433-2439.

31. NEJATI A, et al. Pain management via ultrasound-guided nerve block in emergency department; a case series study. Emergency (Tehran, Iran), 2017; 5(1): 12.

32. RANDALL A, et al. Fascia iliaca compartment block: a nurse-led initiative for preoperative pain management in patients with a fractured neck of femur. Journal of Orthopedic Nursing, 2008; 12(2): 69-74.

33. RITCEY B, et al. Regional nerve blocks for hip and femoral neck fractures in the emergency department: a systematic review. Canadian Journal of Emergency Medicine, 2016; 18(1): 37-47.

34. ROBACK MG, et al. Adverse events associated with procedural sedation and analgesia in a pediatric emergency department: a comparison of common parenteral drugs. Academic Emergency Medicine, 2005; 12(6): 508-513.

35. STONE MB, et al. Ultrasound-guided supraclavicular block for the treatment of upper extremity fractures, dislocations. The American journal of emergency medicine, 2007; 25(4): 472-475.

36. STONE MB, et al. Ultrasound-guided supraclavicular brachial plexus nerve block vs procedural sedation for the treatment of upper extremity emergencies. The American journal of emergency medicine, 2008; 26(6): 706-710.

37. TEZEL O, et al. A comparison of suprascapular nerve block and procedural sedation analgesia in shoulder dislocation reduction. The American journal of emergency medicine, 2014; 32(6): 549-552.

38. TURNER AL, et al. Impact of ultrasound-guided femoral nerve blocks in the pediatric emergency department. Pediatric emergency care, 2014; 30(4): 227-229.

39. WROE $P$, et al. Ultrasound-guided forearm nerve blocks for hand blast injuries: case series and multidisciplinary protocol. The American journal of emergency medicine, 2016; 34(9): 1895-1897. 